Article

\title{
Social Risk Dissociates Social Network Structure across Lateralized Behaviors in Spider Monkeys
}

\author{
Emily R. Boeving *(1) and Eliza L. Nelson \\ Department of Psychology, Florida International University, Miami, FL, 33199, USA; elnelson@fiu.edu \\ * Correspondence: eboev001@fiu.edu; Tel.: +1-305-348-4032
}

Received: 1 August 2018; Accepted: 6 September 2018; Published: 9 September 2018

\begin{abstract}
Reports of lateralized behavior are widespread, although the majority of findings have focused on the visual or motor domains. Less is known about laterality with regards to the social domain. We previously observed a left-side bias in two social affiliative behaviors-embrace and face-embrace-in captive Colombian spider monkeys (Ateles fusciceps rufiventris). Here we applied social network analysis to laterality for the first time. Our findings suggest that laterality influences social structure in spider monkeys with structural differences between networks based on direction of behavioral bias and social interaction type. We attribute these network differences to a graded spectrum of social risk comprised of three dimensions.
\end{abstract}

Keywords: social networks; laterality; social behavior; spider monkey; risk; social interaction

\section{Introduction}

Reports of lateralized behavior are widespread, particularly in the visual and motor domains [1,2]. Decades of research has led to the general consensus that behavioral lateralization is subserved by asymmetric brain function. These brain-behavior asymmetries may serve to streamline neurobiological processes, thereby increasing behavioral efficiency in unpredictable or arousing situations, such as social interactions $[3,4]$. Thus, laterality may be particularly advantageous in gregarious species such as primates.

In a recent synthesis of prior research, Rogers and Vallortigara [1] linked left biases in social behavior to the right hemisphere as a general pattern of lateralization in vertebrates. However, we later showed that not all social behaviors are associated with this pattern of laterality [5]. Specifically, we found that two variations of embracing, but not grooming, were lateralized in Colombian spider monkeys. We argued that the differences in lateralization in social affiliative behaviors were due to the social dynamic in which these behaviors occurred, with grooming considered a low-stakes routine state while embraces were high-stakes risky events. In this study, we focused on assessing the behavioral patterns among individuals within a group, and did not take into account the relational patterns of the group as a whole (e.g., interaction history). While consistent with other laterality investigators, this reductionist approach does not capture the true dynamics of a social system, begging the question: does laterality influence social structure?

Spider monkeys are one of a handful of primates living in fission-fusion [6], a social dynamic defined by separations and reunions. Embraces are a contact greeting gesture that occur at the time of reunions in spider monkeys [7]. In the standard embrace, the hands are wrapped around the body and the face is placed along the trunk [7,8]. A variation is the face-embrace, in which faces touch [5]. Fission-fusion is characterized by marked unpredictability and low social cohesion compared with species that have a known stable hierarchy, cohesive social groups, and low variability in interactive exchanges $[9,10]$. With these differences in mind, social interactions within species living in fission-fusion may consist of a level of risk unlike that experienced in other social dynamics, 
and laterality may play a role in negotiating this risk [2]. In general, social behavior in fission-fusion species is remarkably multi-dimensional, and can be difficult to tease apart.

One method for teasing apart complex social systems is social network analysis [11], a concept with roots in the mathematical field of graph theory. Social network analysis is a tool used to compute and visualize structural relationships in relational data. There is a long history of applying network analysis in the study of sociality in primates (for a review, see [12]) and other species [13]. Yet social network analysis has never been applied in the area of behavioral laterality. Network analysis alone has the unique ability to characterize and mathematically represent global inter-connected elements [14]. Within behavioral laterality, network level information may provide a more sophisticated method to examine topological patterns that represent potential advantages of laterality for behavior, and to accurately depict the multi-dimensional nature of social interaction.

As our primary objective, we leveraged social network analysis in the dataset reported by Boeving, Belnap and Nelson [5] to examine whether similarly lateralized behaviors (i.e., embrace and face-embrace) also have similar network structures, and we predicted that these networks would not differ. In our secondary objective, we examined social networks based on direction of laterality (i.e., left or right) regardless of behavior type by pooling embrace and face-embrace into an affiliative category. We hypothesized that laterality would influence network structure, and we predicted that global left and right affiliative networks would diverge. Finally, we examined the influence of both direction of laterality and behavior type on social network structure by creating four sub-networks of left embrace, left face-embrace, right embrace, and right face-embrace. We hypothesized that laterality, but not behavior type, would alter network structure. We predicted that the left sub-networks would differ from the right sub-networks, but that sub-networks within a behavior (i.e., embrace or face-embrace) would not differ.

\section{Materials and Methods}

\subsection{Social Network Construction from Live Coded Behavior}

We constructed social networks from live coded behavioral observations of 15 captive Colombian spider monkeys (Ateles fusciceps rufiventris). Portions of these data were previously reported in Boeving, Belnap and Nelson [5]. To briefly summarize, $186 \mathrm{~h}$ of data were captured between May and August 2015 using the Animal Behaviour Pro mobile iOS application on apple iPod 5th generation [15]. The application was programmed with information about the individual monkeys to capture initiators and receivers of embrace and face-embrace with the modifier set as side (i.e., left or right positioning). Left or right was recorded with reference to the positioning of the faces regardless of whether there was contact or not. Directionality was not determined by any positioning of the limbs. Data were collected using the continuous sampling method, and ad libitum recording method $[16,17]$ so that all occurrences of the target behaviors could be captured across three equally distributed time periods throughout the day to avoid disruptions due to husbandry procedures. The DuMond Conservancy Institutional Animal Care and Use Committee approved the research, and the study was conducted in accordance with the laws of the United States. The research adhered to the American Society of Primatologists (ASP) Principles for the Ethical Treatment of Non-Human Primates.

\subsection{Social Network Analysis}

We utilized social network analysis as the computational method to investigate potential structural differences within all networks. Networks were computed and visualized in Cytoscape (http:/ /www.cytoscape.com) (Version 3.4.0; [18]), an open source software project for modeling interaction networks. The network metric of degree centrality, which provides a composite score from the in-degree value (i.e., interactions directed towards a monkey) and out-degree value (i.e., interactions directed by a monkey to others), was examined because this metric quantifies the number of edges 
(i.e., social interactions) shared between nodes (i.e., monkeys). The degree centrality of node $(v)$ for a given graph $(G)=(\mathcal{V}, E)$ with $|\mathcal{V}|$ nodes and $|E|$ edges defined as:

$$
C_{D}(v)=\operatorname{deg}(v)
$$

Using the metric degree centrality, the total number of interactions for each individual was computed where monkeys with the most connected interactions (initiated or received) were positioned in the center of the graph and monkeys with fewer connected interactions were positioned along the perimeter. Within Cytoscape, we used a variant of the "Kamada-Kawai Algorithm," a spring-embedded algorithm that forces connected nodes together while also forcing disconnected nodes away from the center [19]. We constructed weighted networks because this method is best suited for graphically representing the variation in social bonds [20,21]. All edges were weighted based on frequency of interaction with thicker edges denoting more interactions and thinner edges denoting fewer interactions. Node size denotes variation in rank of degree centrality where larger nodes indicate higher values of degree centrality and smaller nodes indicate lower values of degree centrality.

\subsection{Statistical Analysis}

To examine whether similarly lateralized behaviors (i.e., embrace and face-embrace) have similar network structures, we first pooled frequency data from each behavior separately regardless of side to create global embrace and global face-embrace networks. To investigate the potential effect of laterality on social network structure, we then pooled affiliative frequency data according to side of positioning to create global left affiliative and global right affiliative networks. Finally, we examined the effect of laterality within each type of embrace by constructing four direction $x$ behavior networks: left embrace, right embrace, left face-embrace, and right face-embrace. $t$-Tests and ANOVA with post hoc comparisons were used to compare the resulting networks.

\section{Results}

A total of 1623 social interactions were examined. Of these, 1270 were embraces and 353 were face-embraces, corresponding to 1227 left affiliative and 396 right affiliative interactions. Individual raw frequency scores for each behavior are reported in Table A1. Four juveniles were excluded from further analysis due to multiple zero values for out-degree, which we suggest is age-related and would not accurately portray degree centrality in the spider monkey group. Network degree centrality values for the global comparisons can be found in Table 1. Unpaired $t$-tests found a significant difference in degree centrality between the global embrace and face-embrace networks $(t(28)=3.43, p<0.01$, $d=1.296$; Figure A1), and a significant difference in degree centrality between the global left and right affiliative networks $(t(20)=3.92, p<0.001, d=1.753$; Figure A2). There was no sex difference in the global left affiliative, global right affiliative, or global embrace networks (all $p>0.05$ ). However, there was a sex difference in the face-embrace network such that females initiated the face-embrace behavior more than males, and males received more of these interactions compared to females $(F(1,13)=4.82$, $\left.p<0.05, \eta^{2}=0.270\right)$. To further examine structural differences between embrace and face-embrace within the context of laterality, we examined the four sub-networks (left embrace, right embrace, left face-embrace, right face-embrace). ANOVA revealed a significant difference in degree centrality among the sub-networks $\left(F(3,40)=20.72, p<0.001, \eta^{2}=0.608\right.$; Figure 1). Post hoc analyses found that each sub-network was different from the others (all $p<0.05)$. 
Table 1. Individual degree centrality values.

\begin{tabular}{cccccc}
\hline Monkey & Sex & Left Affiliative & Right Affiliative & Embrace & Face-Embrace \\
\hline Bon Jovi (Bon) & M & 202 & 57 & 214 & 62 \\
Butch (Bu) & M & 294 & 82 & 263 & 128 \\
Carmelita (Carm) & F & 76 & 25 & 82 & 24 \\
Cleo & F & 208 & 62 & 208 & 73 \\
CJ & F & 108 & 32 & 123 & 19 \\
Dusky (Dusk) & F & 164 & 46 & 191 & 31 \\
Mason (Mas) & M & 372 & 104 & 342 & 141 \\
Mints (Min) & F & 79 & 38 & 136 & 4 \\
Molly (Mol) & F & 94 & 25 & 110 & 15 \\
Sunday (Sun) & M & 261 & 101 & 296 & 83 \\
Uva & M & 386 & 144 & 445 & 121 \\
\hline
\end{tabular}

$\mathrm{M}=$ Male, $\mathrm{F}=$ Female. The higher the degree centrality value, the more highly connected a monkey is to others.

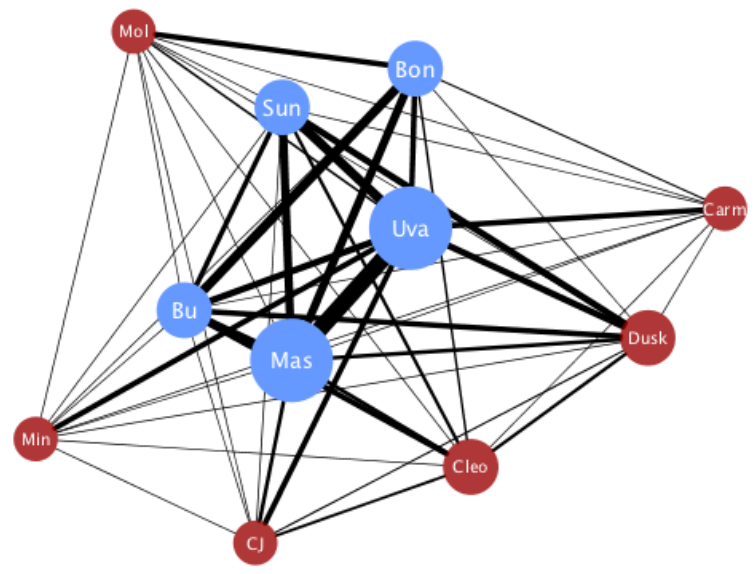

(A)

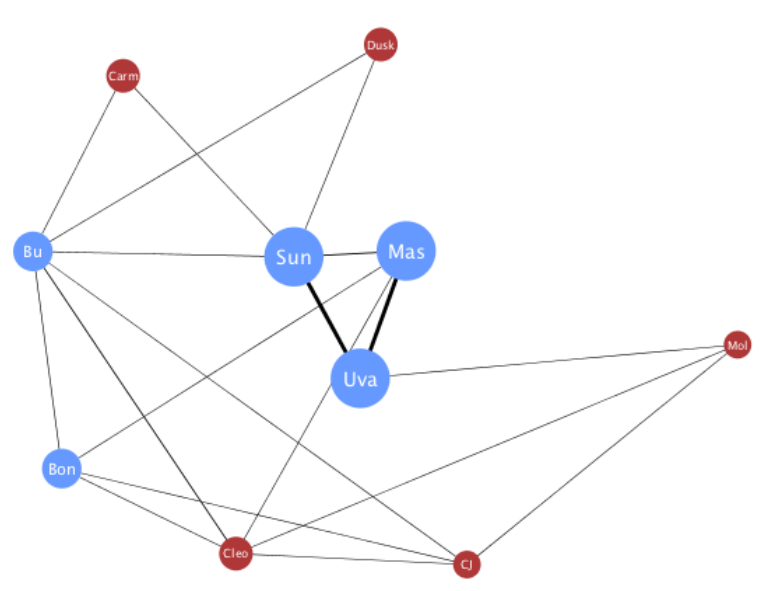

(C)

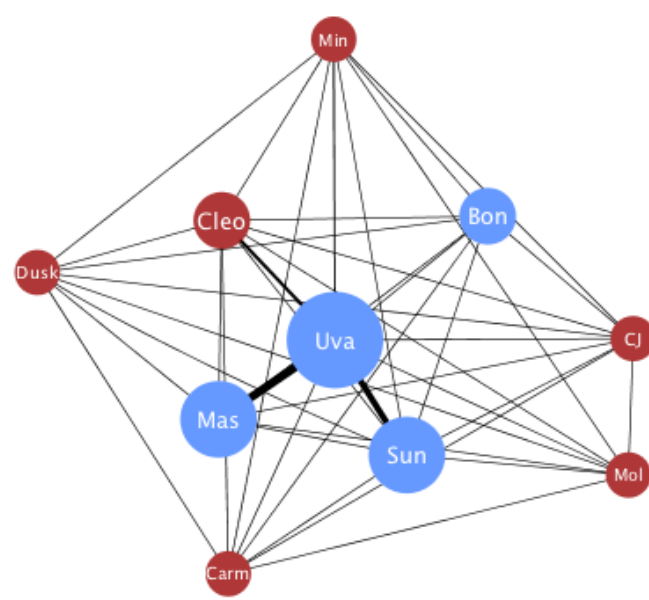

(B)

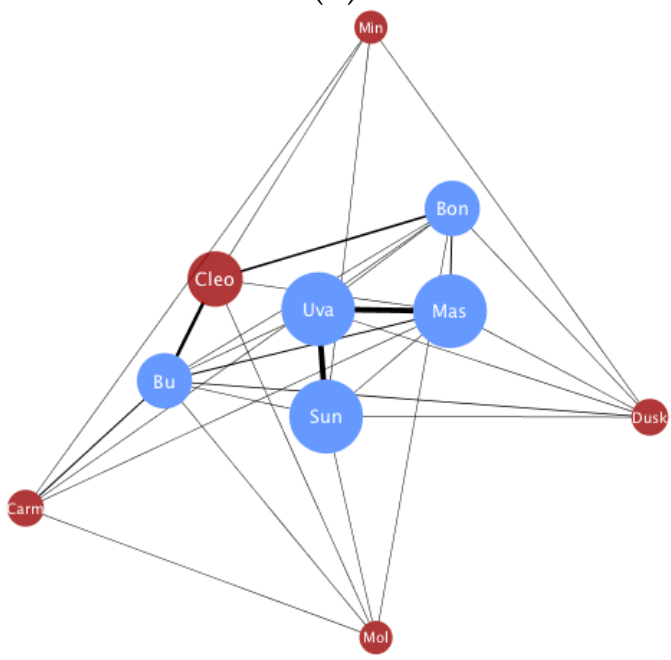

(D)

Figure 1. Clockwise from top left: (A) Left embrace; (B) Right embrace; (C) Left face-embrace; and (D) Right face-embrace. Networks are ordered on social risk index (see text for details). Red denotes females, and blue denotes males. Nodes are weighted such that the larger the node, the higher the degree centrality. Edges are weighted such that thickness denotes frequency of interactions. 


\section{Discussion}

The primary objective of this study was to examine if behaviors with similar patterns of behavioral laterality would also have similar social network structures. We examined the social affiliative behaviors, embrace and face-embrace, which we previously have shown to be left lateralized in spider monkey behavior [5]. Contrary to our predictions, we found that the network for embrace was structurally different from that of face-embrace. We then explored our secondary objective examining whether the side with which the social affiliative behaviors were performed had an effect on network structure. Here our results confirmed our prediction that the global left affiliative network was structurally different from the global right affiliative network. Finally, our analysis of sub-networks parsing direction within each behavior partially supported our prediction. All four sub-networks were different from each other, suggesting an interaction between laterality and behavior type. We discuss these differences in social network structure in the context of three dimensions of social risk.

The concept of risk is often described in the non-human primate literature in the context of risk of aggression from neighboring groups [22], predation [23], and loss of resources [24], all of which are typical challenges for species living in the wild. Rebecchini et al. [25] first identified embracing as a component of risk in spider monkeys, and Boeving, Belnap and Nelson [5] suggested that embrace risk may be graded according to the type of physical contact with face-embrace having higher risk given the close placement of the faces. By comparison, embrace is lower risk because the faces do not touch. Here, we label this type of risk contact risk. Although embrace and face-embrace have a similar left behavioral lateralization pattern, the finding that they do not have similar network structures supports the conclusion that these behaviors are related but distinct. The graphical representation of the embrace network conveys the robustness of this behavior (Figure A1A). Specifically, most individuals engaged in embracing, and with high frequencies, yielding a network graph with most monkeys having high values for degree centrality. Overall, this pattern indicates strong cohesion in the embrace network. In contrast, the face-embrace network depicts interactive patterns in which only a few males were strongly bonded (Figure A1B). When in-degree and out-degree were examined, both males and females initiated and received within the embrace network, but there was a significant difference in the face-embrace network where females initiated more face-embrace and males received more of this behavior. This sex difference is notable because aggression towards females from male spider monkeys is a known pattern [26], making the social lives of female spider monkeys especially risky. In captivity, intra-group aggression is an important consideration given that wild female spider monkeys emigrate from their natal group [26,27]. We envisioned the face-embrace to be the riskier of the two embraces given the close face contact. Yet, with the known pattern of aggression towards females in mind, our social network analysis points to a second aspect of social risk within the face-embrace: partner risk. Social risk in relation to sex roles has been widely discussed in the human literature. For example, female sexual risk taking within certain communities is associated with greater risk of male aggression towards them $[28,29]$. Contact and partner variables have also been examined in the literature on social touch laterality in human kissing [30-34] and embracing [35,36], although these studies have not framed their findings in the context of risk, which may be an avenue in the future to connect these two streams of research.

A third type of risk identified by our network analyses is laterality risk. This dimension of risk was informed by our analyses that identified a structural difference between the global left affiliative and global right affiliative networks. In the left affiliative network, several monkeys were central. In contrast, the right affiliative network had a significantly different architecture in which fewer monkeys were central to the network, and in which the behavior occurred less frequently. Previous work has suggested that the right hemisphere plays an important role in the monitoring and detection of uncertain events in the environment, while the left hemisphere is more involved in routine behavior [2]. This role differentiation between hemispheres is particularly relevant when considering the positioning of the body for embrace and face-embrace. Specifically, if the functional split between hemispheres is correct, then positioning others on the right side for either behavior would 
be risky. Moreover, face-embrace would be especially risky given the close contact of the face coupled with the hypothesized decrease in ability for social monitoring when engaging others on the right side. It would thus be advantageous to position conspecifics on the left side given the hypothesized neural processing benefit. In line with this hypothesis, the structure of the left lateralized affiliative network pattern can be characterized as a highly cohesive network where all monkeys engaged in the behavior, and engaged frequently (Figure A2A). In contrast, the right lateralized network was lower in cohesion; engagement occurred less frequently, with only a few monkeys reaching high values of degree centrality (Figure A2B). Although not recorded in this study, capturing the sequence of behaviors that follow these risky interactions would further test this theory, and is a goal for future work.

Although we collected data over a four-month period, one limitation of this study is that we were not able to assess the stability of these networks over time. Juvenile data were excluded from analyses due to the low frequency of engagement in the behaviors we examined. However, we would expect this pattern to change as individuals mature and develop social bonds. The novel application of social network analysis could quantify this process, not only in primates, but other highly social species. Moreover, here we have utilized a between-networks approach based on our research question, but a within-networks approach across two or more timepoints could provide information about how an individual's position in a network changes as a function of development. A developmental network approach would also broaden our knowledge of the factors that contribute to the emergence of social laterality and its function.

Taken together, the structural differences between the four sub-networks confirmed a graded spectrum of social risk in spider monkeys along the three dimensions of risk: contact, partner, and laterality (Table 2). The sub-network with the lowest risk (i.e., left embrace) had the most participation and strongest cohesion, whereas the sub-network with the highest risk (i.e., right face-embrace) had the least participation and was the most disjointed of the networks indicating low cohesion (Figure 1). To answer our original question posed in the introduction, these findings suggest that laterality influences social structure. However, we acknowledge that social structure may also influence laterality, or that the relationship is bidirectional. Future work using longitudinal designs may address this point. Additional studies should also aim to include network analyses of other behavioral domains that could be related to laterality, such as cognition and motor skill. In conclusion, social network analysis is an exciting new avenue for characterizing brain-behavior relationships. In using this unique computational method to elucidate factors that drive global differences in social network topology, we advance our understanding of laterality within a social framework.

Table 2. Dimensions of social risk.

\begin{tabular}{ccccc}
\hline Behavior & Laterality & Contact & Partner & Risk Index \\
\hline Left Embrace & Low & Low & Low & Lowest \\
Right Embrace & High & Low & Low & Mild \\
Left Face-Embrace & Low & High & High & Moderate \\
Right & High & High & High & Highest \\
Face-Embrace & & & & \\
\hline
\end{tabular}

See text for details.

Author Contributions: Conceptualization, E.R.B. and E.L.N.; Analysis, E.R.B.; Writing—original draft, E.R.B.; Writing-review \& editing, E.R.B. and E.L.N.

Funding: This research received no external funding.

Acknowledgments: We thank Monkey Jungle for supporting this project and members of the HANDS Lab for their assistance with data collection, and Starlie Belnap for her input on the statistical analysis. Alyssa Seidler provided the drawings in the graphical abstract. This is DuMond Conservancy publication no. 60.

Conflicts of Interest: The authors declare no conflict of interest. 


\section{Appendix}

Table A1. Individual Raw Frequency Scores.

\begin{tabular}{cccc}
\hline Monkey & Sex & Embrace & Face-Embrace \\
\hline Bon Jovi (Bon) & M & 92 & 16 \\
Butch (Bu) & M & 107 & 39 \\
Carmelita (Carm) & F & 36 & 17 \\
Cleo & F & 126 & 63 \\
CJ & F & 78 & 14 \\
Dusky (Dusk) & F & 92 & 27 \\
Mason (Mas) & M & 181 & 61 \\
Mints (Min) & F & 47 & 2 \\
Molly (Mol) & F & 81 & 11 \\
Sunday (Sun) & M & 151 & 22 \\
Uva & M & 198 & 80
\end{tabular}

$\mathrm{M}=$ Male, $\mathrm{F}=$ = Female. Frequency is summed across interactions where the monkey initiated or received the behavior.

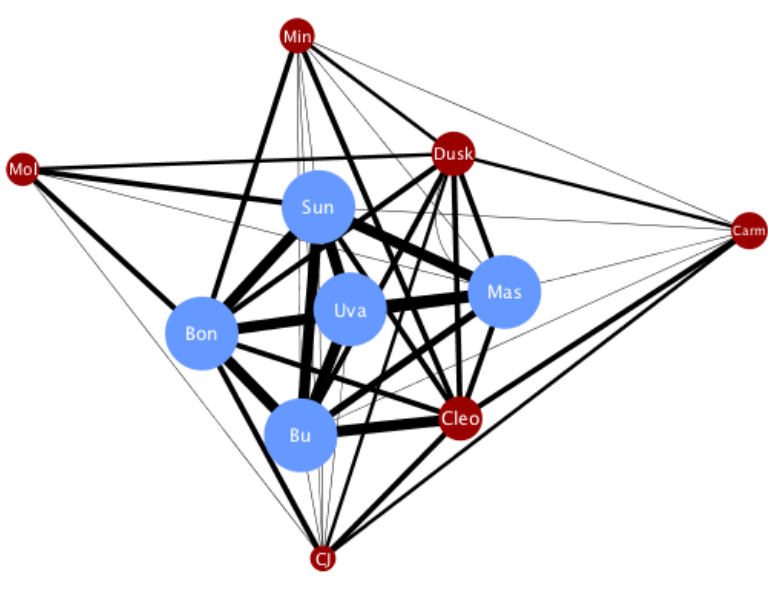

(A)

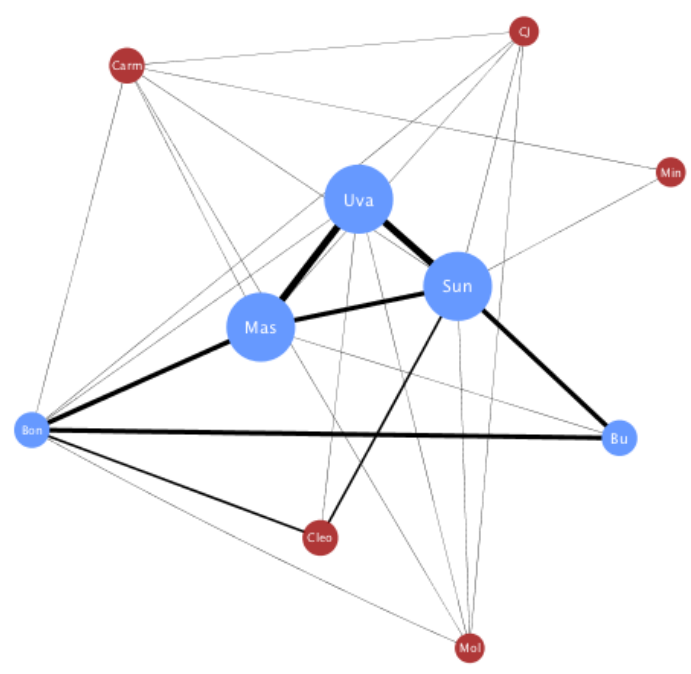

(B)

Figure A1. Global embrace and global face-embrace networks differ.

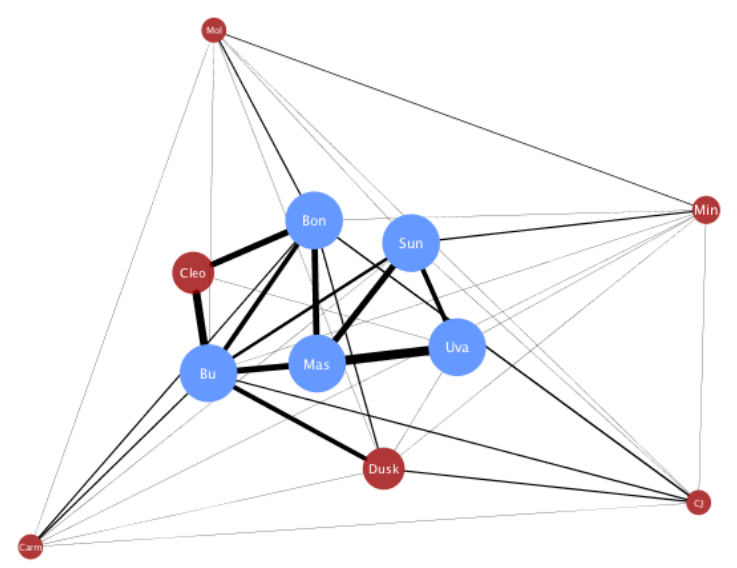

(A)

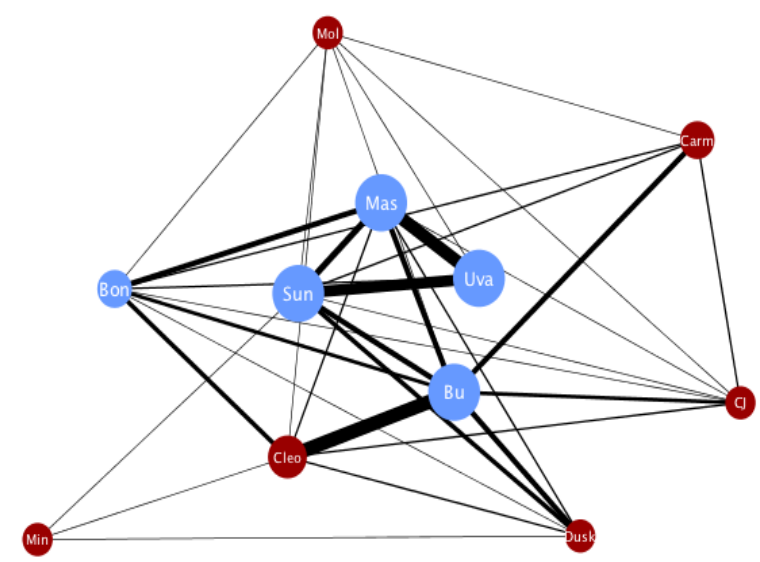

(B)

Figure A2. Global left affiliative and global right affiliative networks differ. Red denotes females, and blue denotes males. Nodes are weighted such that the larger the node, the higher the degree centrality. Edges are weighted such that thickness denotes frequency of interactions. 


\section{References}

1. Rogers, L.J.; Vallortigara, G. When and why did brains break symmetry? Symmetry 2015, 7, $2181-2194$. [CrossRef]

2. MacNeilage, P.F.; Rogers, L.J.; Vallortigara, G. Origins of the left \& right brain. Sci. Am. 2009, 301, 60-67. [PubMed]

3. Rogers, L.J.; Vallortigara, G.; Andrew, R.J. Divided Brains: The Biology and Behaviour of Brain Asymmetries; Cambridge University Press: Cambridge, UK, 2013.

4. Vallortigara, G.; Rogers, L.J. Survival with an asymmetrical brain: Advantages and disadvantages of cerebral lateralization. Behav. Brain Sci. 2005, 28, 575-588. [CrossRef] [PubMed]

5. Boeving, E.R.; Belnap, S.C.; Nelson, E.L. Embraces are lateralized in spider monkeys (Ateles fusciceps rufiventris). Am. J. Primatol. 2017, 79, e22654. [CrossRef] [PubMed]

6. Aguilar-Melo, A.R.; Calmé, S.; Smith-Aguilar, S.E.; Ramos-Fernandez, G. Fission-fusion dynamics as a temporally and spatially flexible behavioral strategy in spider monkeys. Behav. Ecol. Sociobiol. 2018, 72, 150. [CrossRef]

7. Schaffner, C.M.; Aureli, F. Embraces and grooming in captive spider monkeys. Int. J. Primatol. 2005, 26, 1093-1106. [CrossRef]

8. Eisenberg, J.F. Communication mechanisms and social integration in the black spider monkey, ateles fusciceps robustus, and related species. Smithson. Contrib. Zool. 1976, 213, 1-108. [CrossRef]

9. Aureli, F.; Schaffner, C.M.; Boesch, C.; Bearder, S.K.; Call, J.; Chapman, C.A.; Connor, R.; Di Fiore, A.; Dunbar, R.I.; Henzi, S.P. Fission-fusion dynamics. Curr. Anthropol. 2008, 49, 627-654. [CrossRef]

10. Ramos-Fernandez, G.; King, A.J.; Beehner, J.C.; Bergman, T.J.; Crofoot, M.C.; Di Fiore, A.; Lehmann, J.; Schaffner, C.M.; Snyder-Mackler, N.; Zuberbühler, K. Quantifying uncertainty due to fission-fusion dynamics as a component of social complexity. Proc. R. Soc. B 2018, 285, 20180532. [CrossRef] [PubMed]

11. Sueur, C.; Jacobs, A.; Amblard, F.; Petit, O.; King, A.J. How can social network analysis improve the study of primate behavior? Am. J. Primatol. 2011, 73, 703-719. [CrossRef] [PubMed]

12. Brent, L.J.; Lehmann, J.; Ramos-Fernández, G. Social network analysis in the study of nonhuman primates: A historical perspective. A. J. Primatol. 2011, 73, 720-730. [CrossRef] [PubMed]

13. Wey, T.; Blumstein, D.T.; Shen, W.; Jordán, F. Social network analysis of animal behaviour: A promising tool for the study of sociality. Anim. Behav. 2008, 75, 333-344. [CrossRef]

14. Sporns, O. The human connectome: A complex network. Ann. N. Y. Acad. Sci. 2011, 1224, 109-125. [CrossRef] [PubMed]

15. Newton-Fisher, N.E. Animal Behaviour Pro: V1; Apple: Canterbury, UK, 2012.

16. Martin, P.; Bateson, P.P.G.; Bateson, P. Measuring Behaviour: An Introductory Guide; Cambridge University Press: Cambridge, UK, 1993.

17. Altmann, J. Observational study of behavior: Sampling methods. Behaviour 1974, 49, 227-266. [CrossRef] [PubMed]

18. Shannon, P.; Markiel, A.; Ozier, O.; Baliga, N.S.; Wang, J.T.; Ramage, D.; Amin, N.; Schwikowski, B.; Ideker, T. Cytoscape: A software environment for integrated models of biomolecular interaction networks. Genome Res. 2003, 13, 2498-2504. [CrossRef] [PubMed]

19. Kamada, T.; Kawai, S. An algorithm for drawing general undirected graphs. Inf. Process. Lett. 1989, 31, 7-15. [CrossRef]

20. Kerth, G.; Perony, N.; Schweitzer, F. Bats are able to maintain long-term social relationships despite the high fission-fusion dynamics of their groups. Proc. R. Soc. Lond. B. Biol. Sci. 2011, 278, 2761-2767. [CrossRef] [PubMed]

21. Voelkl, B.; Kasper, C.; Schwab, C. Network measures for dyadic interactions: Stability and reliability. Am. J. Primatol. 2011, 73, 731-740. [CrossRef] [PubMed]

22. Wrangham, R.; Crofoot, M.; Lundy, R.; Gilby, I. Use of overlap zones among group-living primates: A test of the risk hypothesis. Behaviour 2007, 144, 1599-1619. [CrossRef]

23. Hill, R.; Lee, P. Predation risk as an influence on group size in cercopithecoid primates: Implications for social structure. J. Zool. 1998, 245, 447-456. [CrossRef]

24. Jernvall, J.; Wright, P.C. Diversity components of impending primate extinctions. Proc. Natl. Acad. Sci. USA 1998, 95, 11279-11283. [CrossRef] [PubMed] 
25. Rebecchini, L.; Schaffner, C.M.; Aureli, F. Risk is a component of social relationships in spider monkeys. Ethology 2011, 117, 691-699. [CrossRef]

26. Fedigan, L.M.; Baxter, M.J. Sex differences and social organization in free-ranging spider monkeys (Ateles geoffroyi). Primates 1984, 25, 279-294. [CrossRef]

27. Link, A.; Milich, K.; Di Fiore, A. Demography and life history of a group of white-bellied spider monkeys (Ateles belzebuth) in western amazonia. Am. J. Primatol. 2018, e22899. [CrossRef] [PubMed]

28. Campbell, J.C.; Baty, M.; Ghandour, R.M.; Stockman, J.K.; Francisco, L.; Wagman, J. The intersection of intimate partner violence against women and hiv/aids: A review. Int. J. Inj. Control Saf. Promot. 2008, 15, 221-231. [CrossRef] [PubMed]

29. Jewkes, R.K.; Levin, J.B.; Penn-Kekana, L.A. Gender inequalities, intimate partner violence and hiv preventive practices: Findings of a south african cross-sectional study. Soc. Sci. Med. 2003, 56, 125-134. [CrossRef]

30. Güntürkün, O. Human behaviour: Adult persistence of head-turning asymmetry. Nature 2003, 421, 711. [CrossRef] [PubMed]

31. Chapelain, A.; Pimbert, P.; Aube, L.; Perrocheau, O.; Debunne, G.; Bellido, A.; Blois-Heulin, C. Can population-level laterality stem from social pressures? Evidence from cheek kissing in humans. PLoS ONE 2015, 10, e0124477. [CrossRef] [PubMed]

32. Ocklenburg, S.; Güntürkün, O. Head-turning asymmetries during kissing and their association with lateral preference. Laterality 2009, 14, 79-85. [CrossRef] [PubMed]

33. Sedgewick, J.R.; Elias, L.J. Family matters: Directionality of turning bias while kissing is modulated by context. Laterality Asymmetries Body Brain Cogn. 2016, 21, 662-671. [CrossRef] [PubMed]

34. Van der Kamp, J.; Canal-Bruland, R. Kissing right? On the consistency of the head-turning bias in kissing. Laterality 2011, 16, 257-267. [CrossRef] [PubMed]

35. Packheiser, J.; Rook, N.; Dursun, Z.; Mesenhöller, J.; Wenglorz, A.; Güntürkün, O.; Ocklenburg, S. Embracing your emotions: Affective state impacts lateralisation of human embraces. Psychol. Res. 2018, 1-11. [CrossRef] [PubMed]

36. Turnbull, O.; Stein, L.; Lucas, M. Lateral preferences in adult embracing: A test of the "hemispheric asymmetry" theory of infant cradling. J. Genet. Psychol. 1995, 156, 17-21. [CrossRef]

(C) 2018 by the authors. Licensee MDPI, Basel, Switzerland. This article is an open access article distributed under the terms and conditions of the Creative Commons Attribution (CC BY) license (http://creativecommons.org/licenses/by/4.0/). 